\title{
NOTE ON THE ANNULUS CONJECTURE ${ }^{1}$
}

\author{
W. A. LABACH
}

We give here a partial solution of the annulus conjecture which includes all known partial solutions except those assuming a condition equivalent to the annulus conjecture.

Lipschitz (or $C^{1 / 2}$ ) manifolds and maps are defined in [4]. By a Lipschitz submanifold of a Euclidean space we mean a $C^{r}$ submanifold in the sense of [3] with $r=\frac{1}{2}$. A 1-1 Lipschitz mapping of one metric space on to another is said to be regular if and only if its inverse is Lipschitz.

THEOREM. An $n$-dimensional annular region in $R^{n}$ is an annulus if each of its bounding spheres contains a non-empty open subset which is a Lipschitz submanifold of $R^{n}$.

Proof. Let $S$ be a bounding sphere of our annular region. We identify $S \times(-1,1)$ with the standard open $n$-annulus $S^{n-1} \times(-1,1)$ by a homeomorphism $(x, t) \rightarrow(f(x), t)$ where $x \in S, t \in(-1,1)$, and $f: S \rightarrow S^{n-1}$ is a homeomorphism. It follows from our hypothesis that there is a regular Lipschitz homeomorphism $g: U \times(-1,1) \rightarrow R^{n}$ such that $g(x, 0)=x$ for all $x \in U$. Let $V \subset U$ be a nonempty open subset of $S$ such that $\bar{V} \subset U$. Then, by Brown [1], there is a mapping $h: S \times(-1,1) \rightarrow R^{n}$ such that $h(x, 0)=x$ for all $x \in S$ and $h\left(x^{\prime}, t\right)$ $=g\left(x^{\prime}, t\right)$ for all $x^{\prime} \in V, t \in(-1,1)$. By Rademacher's theorem [5, p. 272] and the chain rule, the mapping $h \mid V \times(-1,1)$ is differentiable with nonvanishing Jacobian almost everywhere. By Kirby [2], our annular region is an annulus.

\section{REFERENCES}

1. M. Brown, Locally flat embeddings of topological manifolds, Ann. of Math. $\mathbf{7 5}$ (1962), 331-341.

2. R. C. Kirby, On the annulus conjecture, Proc. Amer. Math. Soc. 17 (1966), 178-185.

3. J. Munkres, Elementary differential topology, Princeton Univ. Press, Princeton, N. J., 1963.

4. J. H. C. Whitehead, Manifolds with transverse fields in Euclidean space, Ann. of Math. 73 (1961), 154-212.

5. H. Whitney, Geometric integration theory, Princeton Univ. Press, Princeton, N. J., 1957.

\section{Florida State University}

Received by the editors September 26, 1966.

1 This work was partially supported by the National Science Foundation under Grant G-5458. 